\title{
Fine Phase Resolved X-ray Spectroscopy of the Crab Pulsar with BeppoSAX
}

\author{
E. Massaro, M. Litterio \\ Istituto Astronomico, Università "La Sapienza", Roma, Italy \\ G. Cusumano, T. Mineo \\ Istituto di Fisica Cosmica e Appl. Informatica, CNR, Palermo, Italy
}

\begin{abstract}
We present the results of a phase resolved spectroscopy of the Crab pulsar in the band $0.1-200 \mathrm{keV}$ based on many observations performed with BeppoSAX. The spectrum was found soft in the main peak and significantly harder in the Interpeak region. This behaviour is explained by means of a two component model.
\end{abstract}

\section{Introduction}

The Italian-Dutch satellite BeppoSAX observed the Crab Nebula and Pulsar in 1996 during the Science Verification Phase (Mineo et al. 1997). From that epoch to the spring of 1999 this source was pointed several times because it is used for the calibration checking of the four Narrow Field Instruments (NFI: LECS, MECS, HPGSPC, PDS). All these observations were used for a very accurate phase resolved spectroscopy over an energy range from about 0.5 up to 200 $\mathrm{keV}$. In this contribution we present the results of the analysis of all BeppoSAX observations summed together.

\section{Observations and Data Reduction}

Phase histograms of the Crab pulsar were evaluated for each NFIs and each pointing period using the standard folding technique, after conversion of the UTC arrival times of all selected events to the Solar System Barycentre using the DE200 ephemeris. We constructed a large set of 300 bin phase histograms for every energy channel of each NFI. The zero phase was fixed at the centre of P1, evaluated by means of gaussian fits; also, we verified that all the pulse profiles had statistically compatible shapes. All the histograms for the same energy channel were then added together.

Spectral analysis was performed adopting a minimum phase interval of 0.0067 and applying the standard response matrices of the four NFIs. In the Interpeak region (Ip), however, where the signal level is low, we used wider phase intervals up to obtain statistical errors on the spectral slopes less than 0.05 . Spectral indices were evaluated after subtraction of the off-pulse level, taken in the phase interval $(0.47-0.77)$. Here we present some MECS and PDS results. 

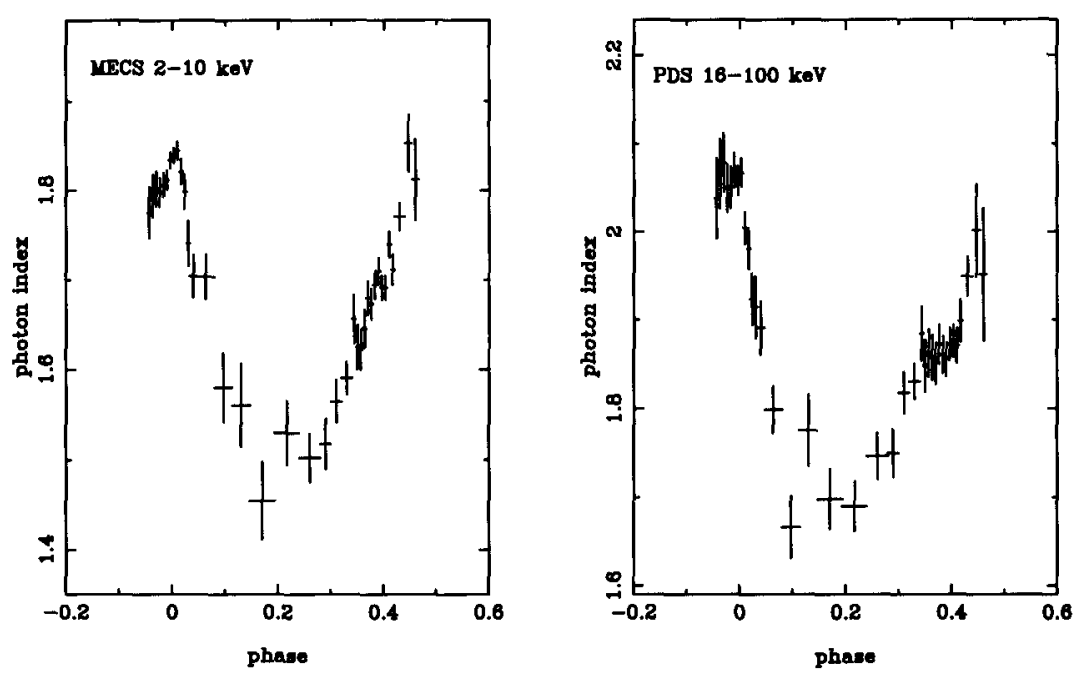

Figure 1. The phase evolution of the photon spectral indices for the MECS and PDS energy ranges.

\section{Results}

The photon spectral indices for single power law fits of the MECS and PDS data are shown in Fig. 1. The same behaviour along the phase is clearly apparent in both plots: the feature with the softest spectrum is $\mathrm{P} 1$, while the middle of the Ip is the hardest with a spectral index difference of about 0.30 , as already found by Pravdo et al. (1997).

This behaviour suggests that the $\mathrm{X}$-ray emission of the Crab pulsar is the sum of two components having different energy and phase distributions. We verified that adding to the optical profile a harder second component, with a roughly triangular shape peaked at the phase 0.4 and a very steep trailing edge, all data in Fig.1 are well reproduced. It is an open problem the understanding the emission mechanisms and the locations in the magnetosphere for both these components. Likely, they could be related to particles accelerated in various possible sites, like polar and outer gaps. In this case, the different spectral shapes could be due to the relative contributions of the radiation from secondary electrons and positrons, originated by primary high energy $\gamma$ rays. A more detailed model is necessary to confirm this hypothesis and to unveil the nature of the two components.

\section{References}

Mineo, T. et al. 1997, A\&A 327, L21

Pravdo, S.H., Angelini, L., Harding, A.K. 1997, Ap.J 491, 808 